Patients with unexplained LGI symptoms who do not meet the criteria for a suspected LGI cancer pathway triaged using FIT-Hb pathway and proforma request form

Indication for FIT-Hb (inclusion criteria)

1 - Aged $\geq 50$ yrs with:

- Change in Bowel Habit OR

- Unexplained Abdominal pain OR

- Unexplained Weight loss alone OR

2- Aged $\geq 60$ yrs with:

- Anaemia (without iron deficiency)

FIT cut-off (Alpha-Labs):

POSITIVE $\geq 10 \mu \mathrm{g} \mathrm{Hb} / \mathrm{g}$ faeces $=$ Referral via $2 \mathrm{WW}$ Pathway

NEGATIVE $<10 \mu \mathrm{g} \mathrm{Hb} / \mathrm{g}$ faeces = Consider URGENT Gastroenterology referral if clinical concerns/persistent symptoms

Methods Prospective data collection (July to December 2018).

Data collected from FIT request forms, endoscopy, pathology, radiology system and clinic correspondence.

Data collected on demographics, indication and adherence to inclusion criteria, result and clinical outcome.

Results 13 GP practices enrolled in pilot.

97 patients underwent FIT-Hb testing $(87 \%$ had complete data).

All patients were $\geq 50$ years (average $70 y, 33 \%$ male).

Inclusion criteria were met in all patients, change in bowel habit (51\%), unexplained abdominal pain (39\%), $16 \% \geq 1$ indication.

FIT-Hb positive in 20 patients (24\%, range $1-93 \mu \mathrm{gHb} / \mathrm{g})$. All patients were referred for $2 \mathrm{WW}$ GI investigation, $75 \%$ colonoscopy, 20\% CT-Colonography, 5\% no investigation to date.

Diagnostic outcomes: Colorectal cancer (2), colonic polyps (3, all low risk polyps), diverticular disease (2), colitis (1)

Despite a negative FIT-Hb result, 2 patients (4\%) ultimately had colonoscopy (1 low risk polyp detected).

No FIT-Hb-negative patients diagnosed with LGI malignancies during the limited follow-up period (- months).

Conclusions Assuming all FIT-Hb tested patients would have been referred to secondary care for lower GI investigation, the data suggests $72 \%$ reduction in outpatient referral and colonoscopy/CTC. No CRC diagnoses were missed by a negative FIT$\mathrm{Hb}$ during the limited follow-up period.

Direct verbal feedback suggests the enrolled GP practices have confidence in the pathway. Prospective data collection continues, but based on the limited pilot data and recent NICE recommendation, a FIT-Hb pathway has been formally commissioned by the Herefordshire CCG.

\section{PTU-078 DOES COMPLEX POLYPS MULTI-DISCIPLINARY TEAM CHANGE PATIENTS' OUTCOME? DISTRICT GENERAL HOSPITAL EXPERIENCE}

Ammar Al-Rifaie*, Dominic Bullas, Mr RaoKhalid Mehmood, Ms Karen Smith, Ashraf Soliman, Elmuhtady Said. Barnsley Hospital NHS Foundation Trust, UK

\subsection{6/gutjnl-2019-BSGAbstracts.437}

Introduction The management of advanced non-cancerous GI polyps can be challenging. Data on the impact of complex polyps Multi-disciplinary Team (ComP MDT) is lacking. Our aim is to evaluate the outcomes of ComP MDT in our hospital.
Methods ComP MDT was established in Barnsley Hospital in April 2018 \&amp held monthly. Data prospectively collected (Apr2018-Jan2019). ComP MDT team included advanced endoscopists, gastroenterologists and colorectal surgeon. All referrals were received internally with an initial plan. The outcome is approved after discussing each case to reach a consensus.

Results 53 cases discussed in 10 meetings. There were 29 males vs 24 females with a mean age of 68.1 years. 46/53 (87\%) were colonic polyps (CP) compared to $7 / 53(13 \%)$ upper GI polyps (UGIP). The most common indications for referring CP were: multiple polyps ranged from -0 polyps (33\%), polyp size ranged 2-0mm (20\%), morphology; flat \& uncertain pit pattern (20\%), difficult colonoscopy; long, loopy $\&$ fixed colons (15\%) \& difficult location (22\%). CP were situated mainly in the rectosigmoid $19 / 46$ (41\%) vs $17 / 46$ (37\%) in the right colon (caecum \& ascending colon).

ComP MDT agreement with initial endoscopist lesion assessment was observed in 41/53 (78\%). However, changes to proposed resection plan were recommended in $21 / 53$ (40\%). The range of SMSA score for CP was -4 . 26/46 $(57 \%)$ were level - polyps. Endoscopic mucosal resection (EMR) was approved and achieved in 25/46 (54\%). Only one patient had complication with mild postpolypectomy bleeding that was managed conservatively as an inpatient. 6/46 (13\%) were referred directly for surgical resection, $3 / 46$ (7\%) referred to tertiary centre, $7 / 46(15 \%)$ were not fit for resection \& 5/46 (11\%) are awaiting EMR. Recommendations for type of bowel preparation, time dedicated to EMR, type of colonoscope \& operating endoscopist were also advised.

Out of all CP reviewed at ComP MDT, two found to be cancerous polyps, one successfully removed surgically (confirmed adenocarcinoma) \& the other managed conservatively.

Polyp type uncertainty was the reason for referring UGIP (5 gastric \& 2 duodenal polyps). One gastric polyp resected successfully, three proven hyperplastic histologically \& one referred for endoscopic ultrasonography. One duodenal polyp was resected $\&$ one directed to cancer pathway.

Conclusion ComP MDT changes the patients' outcome in a significant number of referrals by directing patients to the appropriate intervention. Safe \& successful resection of all advanced polyps which deemed resectable on ComP MDT was achieved. ComP MDT was able to pick up some cancerous lesions that were initially misdiagnosed as benign polyps.

\section{PTU-079 ASSESSING GLUTEN FREE DIET ADHERENCE USING CDAT AND BIAGI QUESTIONNAIRES IN PATIENTS WITH COELIAC DISEASE}

Ms Elisabeth Baggus*, Anupam Rej, Michelle Lau, Peter Mooney, Mr Micheal Rees, Mr William White, Ms Lauren Marks, Marios Hadjivassiliou, David Sanders. Academic Unit Of Gastroenterology, Royal Hallamshire Hospital, Sheffield, UK

\subsection{6/gutjnl-2019-BSGAbstracts.438}

Introduction The gold standard currently for assessing adherence in individuals with coeliac disease is via duodenal biopsies, which is invasive and costly. In view of this, we assessed the utility of the CDAT and Biagi questionnaires for non-invasive assessment of gluten free adherence.

Methods Patients with an established diagnosis of coeliac disease, referred for further evaluation of dietary adherence and disease remission were assessed between January 2016 to December 2018. Patients were prospectively recruited, and 
completed CDAT and Biagi questionnaires, with at least 4 duodenal biopsies taken from D2 in addition to at least one biopsy from the duodenal bulb. The presence (Marsh 3a or above) or absence (Marsh 0-II) of villous atrophy was used to determine the sensitivities of the tests.

Results 151 patients were recruited, 101 females (66.9\%), median age 55.0 years, median duration of GFD of 72.0 months. Table 1 outlines the sensitivity and specificity of the CDAT questionnaire, Biagi questionnaire, IgA-TTG and IgAEMA.

Conclusions The sensitivity of the CDAT questionnaire was not superior to IgA-TTG for predicting villous atrophy in patients with coeliac disease. However, the use of a combination of both Biagi and CDAT had a greater sensitivity than IgA-TTG and IgA-EMA $(\mathrm{p}<0.05)$, but lower specificity $(\mathrm{p}<0.05)$. Duodenal biopsy remains the gold standard, although these scores remain useful tools in the assessment of dietary adherence.

\begin{tabular}{|c|c|c|c|c|}
\hline & $\begin{array}{l}\text { Sensitivity } \\
\%(\mathrm{Cl})\end{array}$ & $\begin{array}{l}\text { Specificity } \\
\%(\mathrm{Cl})\end{array}$ & $\begin{array}{l}\text { Positive predictive } \\
\text { value } \%(\mathrm{Cl})\end{array}$ & $\begin{array}{l}\text { Negative predictive } \\
\text { value } \%(\mathrm{Cl})\end{array}$ \\
\hline CDAT & $\begin{array}{l}52.0(37.6- \\
66.1)\end{array}$ & $\begin{array}{l}69.8(60 .- \\
7.5)\end{array}$ & $40.6(28.8-53.6)$ & $78.6(69.6-85.5)$ \\
\hline Biagi & $\begin{array}{l}22.4 \\
(12 .-7.0)\end{array}$ & $\begin{array}{l}93.1(85.6- \\
97.0)\end{array}$ & $\begin{array}{l}61.1 \\
(36.1-81.7)\end{array}$ & $\begin{array}{l}71.4 \\
(62.8-78.8)\end{array}$ \\
\hline $\begin{array}{l}\text { CDAT \& } \\
\text { Biagi }\end{array}$ & $\begin{array}{l}61.2(46 .- \\
4.8)\end{array}$ & $\begin{array}{l}69.3(60 .- \\
7.2)\end{array}$ & $43.5(35 .-2.0)$ & $82.2(76 .-7.0)$ \\
\hline IgA-TTG & $\begin{array}{l}30.6 \\
(18.7-45.6)\end{array}$ & $\begin{array}{l}91.6(83.6- \\
96.0)\end{array}$ & $\begin{array}{l}65.2 \\
(42.8-82.8)\end{array}$ & $\begin{array}{l}71.9 \\
(62.8-79.5)\end{array}$ \\
\hline IgA-EMA & $\begin{array}{l}34.3(26.7- \\
42.7)\end{array}$ & $\begin{array}{l}92.5(84.8- \\
96.7)\end{array}$ & $68.2(45.1-85.2)$ & $71.9(62.9-79.5)$ \\
\hline
\end{tabular}

\section{PTU-080 SHOULD WE ALL BE LOOKING FOR MARGINAL GAINS IN ENDOSCOPY EFFICIENCY?}

${ }^{1}$ Kathleen Bryce*, ${ }^{1}$ Cheh Kuan Tai, ${ }^{2}$ Sam Murray, ${ }^{1}$ Robert Fearn. ${ }^{1}$ Homerton University Hospital NHS Foundation Trust, London, UK; ${ }^{2}$ North Bristol NHS Trust, Bristol, UK

\subsection{6/gutjnl-2019-BSGAbstracts.439}

Introduction Demand for endoscopy in the UK has doubled in the last 5 years. In 2017, 64\% of units failed to meet suspected cancer targets despite $66 \%$ of units having weekend lists and $27 \%$ outsourcing to external providers ${ }^{1}$. UCL Cancer Collaborative (UCLCC) data showed demand can be met by improving efficiency. This is important in a resource-limited setting. Our Quality Improvement (QI) Project aimed to improve efficiency by improving turnaround time, non-attendance and on-the-day cancellations.

Methods The Endoscopy QI fellow, endoscopy unit manager and Gastroenterology service manager participated in the UCLCC Improvement Programme, and utilised QI methodology. We collected data from electronic patient records and scheduling system.

At baseline, we identified that underutilisation of lists was multifactorial. We introduced a turnaround nurse role to consent patients. Healthcare assistants (HCAS) and nurses were trained in cannulation. As poor bowel preparation contributed to cancellations, we introduced telephone pre-assessment to educate patients. Finally, the administrative team sent text reminders before appointments.

Results At baseline, our unit performed an average of 7.9 points per list, out of a planned 10. On average, 28.5 patients per month had procedures cancelled on the day due to poor bowel prep or inadequate fasting. After the introduction of pre-assessment, it improved to 23.5 per month, saving 5 procedures which would have had to be rearranged. The average points performed improved to 9.3 points per list.

The average DNA rate has improved from $9 \%$ to $7 \%$ after the introduction of text reminders. After the introduction of the turnaround nurse and HCA cannulation, turnaround time reduced from an average of 18 to 9 minutes between procedures. This could save 90 minutes over a 12-point list.

Despite these improvements, only $41.6 \%$ of lists are booked for 1-2 points. Inadequate staffing numbers and late start times are contributing factors. A start time audit showed that only 5 out of 27 lists in a week started within 10 minutes of supposed start times, a target for further cycles of this QI project.

Conclusions Multiple small improvements in efficiency can achieve significant impact on productivity. Interventions focused on turnaround time can reduce underuse of list time. Patient-centred approaches to procedural preparation may reduce squandered appointments. Sustainability of these improvements is difficult to assess in the short term but will be promoted by the continuing QI fellow role and implementation of endoscopy QI champions from the administrative and nursing team.

\section{REFERENCE}

1. Shenbagaraj, L., Thomas-Gibson, S., Stebbing, J., Broughton, R., Dron, M., Johnston, D. et al. (2018) Endoscopy in 2017: A national survey of practice in the UK, Frontline Gastroenterology, pp. 100970. DOI: 10.1136/flgastro-201-00970.

\section{PTU-081 ENHANCING EFFICIENCY IN ENDOSCOPY UNIT USING THE 'TIME AND MOTION' MODEL}

${ }^{1}$ Shivkumar Budihal ${ }^{*},{ }^{1} \mathrm{Ms}$ Isobel Esberger, ${ }^{1} \mathrm{Ms}$ Ann Fertwell, ${ }^{1} \mathrm{Mr}$ Richard Canetti, ${ }^{1,2}$ Adolfo Parra-Blanco, ${ }^{1,2}$ Krish Ragunath. 'Nottingham University Hospitals, Nottingham, UK; ${ }^{2}$ NIHR BRC at Nottingham University Hospitals and University of Nottingham, Nottingham, UK

\subsection{6/gutjnl-2019-BSGAbstracts.440}

Introduction Endoscopy Units throughout the UK are facing unprecedented pressures due to increasing demand with limited capacity. Our aim was to explore strategies to improve our endoscopy unit's efficiency. In order to identify targets for improvement we sought to undertake a 'Time and Motion' study in the endoscopy unit. Methods

The study was conducted at Nottingham City Hospital Endoscopy centre between 13th January 2017 to 10th February 2017. Procedures included were gastroscopy, colonoscopy, sigmoidoscopy, bronchoscopy, therapeutic endoscopy and endoscopic ultrasound. From the time a patient reports to the reception to the time discharge was made the patients journey was mapped using the 'time and motion' principle. As part of this study, healthcare professionals and admission staff were asked to record the patient's journey through endoscopy unit accurately and complete templates which were kept with the patient's notes as they moved through the unit. Separate 